\title{
Non-relativistic quantum theory at finite temperature
}

\author{
Xiang-Yao $\mathrm{Wu}^{a}{ }^{*}$, Bo-Jun Zhang ${ }^{a}$, Xiao-Jing Liu ${ }^{a}$, Si-Qi Zhang ${ }^{a}$ \\ Jing Wang ${ }^{a}, \mathrm{Hong} \mathrm{Li}^{a}$, Nou $\mathrm{Ba}^{a}$, Li Xiao ${ }^{a}$ and Yi-Heng $\mathrm{Wu}^{b}$ \\ a.Institute of Physics, Jilin Normal University, Siping 136000 \\ b. Institute of Physics, Jilin University, Changchun 130012
}

\begin{abstract}
We propose the non-relativistic finite temperature quantum wave equations for a single particle and multiple particles. We give the relation between energy eigenvalues, eigenfunctions, transition frequency and temperature, and obtain some results: (1) when the degeneracies of two energy levels are same, the transition frequency between the two energy levels is unchanged when the temperature is changed. (2) When the degeneracies of two energy levels are different, the variance of transition frequency at two energy levels is direct proportion to temperature difference.
\end{abstract}

PACS: 11.10.Wx, 03.65.-w

Keywords: finite temperature; Quantum theory

\section{Introduction}

Quantum field theory at finite temperature was motivated by the increasing interest in studying the properties of matter under extreme conditions as, for example, at very high temperature or density. The pioneering works joining together the statistical and quantum field theory were developed mainly by Matsubara [1] in a non-relativistic context and, the relativistic case by Fradkin [2]. The finite temperature gauge theories and the problems concerning to the choice of a physical gauge and its dependence was analyzed by Bernard [3], in particular, the free electromagnetic field. Thermodynamics and statistical mechanics are powerful and vastly general tools. A rather fuller review of the necessary statistical mechanics may be found in the book by Fetter and Walecka [4], which also gives a very full account of non-relativistic finite-temperature field theory. Semiclassical series were introduced in quantum mechanics by the pioneering works of Brillouin [5], Kramers [6] and Wentzel [7]. Semiclassical methods for finite temperature field theories [8-10] also remained restricted to derivations of the first term of a semiclassical series [11], even when the problem was reduced to quantum statistical mechanics [12, 13], viewed as field theory at a point.

In thermodynamic, the thermodynamic quantities such as internal energy, free energy, volume, pressure, entropy and so on are effected by temperature, which are described by the first and second laws of thermodynamics. In quantum statistical, it is only considered the impact of temperature on the energy level, particle numbers and probability distribution, and it isn't from the quantum theory including temperature. In non-relativistic quantum theory, the Schrodinger equation doesn't include temperature. Therefore, the full quantum theory and quantum statistical theory should be included temperature. In this paper, we extend the Schrodinger equation from zero temperature to finite temperature. With the finite temperature quantum theory, we can study the affect of temperature on quantum systems.

\section{The free energy of thermodynamic system}

For a system constituting of $N$ particles, the free energy is defined by

$$
F=U-T S,
$$

where $F, U, S$ and $T$ are the free energy, internal energy, entropy and temperature of the system, respectively.

In the system, every particle can be in a series of state, we define $T_{j}^{i}, V_{j}^{i}$ are the kinetic energy and

\footnotetext{
* E-mail: wuxy2066@163.com
} 
potential energy of the $j-t h$ particle in the $i-t h$ state, $V_{j m}^{i}$ is the interaction energy between the $j-t h$ and the $m-t h$ particle in the $i-t h$ state. The microcosmic internal energy is

$$
U^{i}=\sum_{j=1}^{N_{i}}\left(T_{j}^{i}+V_{j}^{i}\right)+\sum_{j, m}^{N_{i}} V_{j m}^{i}
$$

where $N_{i}$ is the particle number of system in the $i-t h$ microscopic state, and $V_{j m}^{i}=0(j=m)$. According to statistical principle, the macroscopic internal energy of system is a statistical average value of its microscopic internal energy. The macroscopic internal energy $U$ is

$$
U=\sum_{i=1}^{M} P_{i} U^{i}=\sum_{i=1}^{M} P_{i}\left(\sum_{j=1}^{N_{i}}\left(T_{j}^{i}+V_{j}^{i}\right)+\sum_{j, m}^{N_{i}} V_{j m}^{i}\right),
$$

where $P_{i}$ is the probability of system in the $i-t h$ microscopic state, and $M$ is the microscopic state number of system.

Defining free energy $f_{j}^{i}$, it is the $j-t h$ particle in the $i-t h$ state, then the system microscopic free energy in the $i-t h$ state is

$$
F^{i}=\sum_{j=1}^{N_{i}} f_{j}^{i}
$$

the macroscopic free energy of system is a statistical average value of its microscopic free energy. The macroscopic free energy $F$ is

$$
F=\sum_{i=1}^{M} P_{i} F^{i}=\sum_{i=1}^{M} \sum_{j=1}^{N_{i}} P_{i} f_{j}^{i},
$$

the microscopic entropy of the $j-t h$ particle in the $i-t h$ state $S_{j}^{i}$ is

$$
S_{j}^{i}=-k_{B} \ln \left(\omega_{j}^{i} p_{j}^{i}\right)
$$

Where $\omega_{j}^{i}$ is the degeneracy of the $j-t h$ particle in the $i-t h$ state, $p_{j}^{i}$ is the probability of the $j-t h$ particle in the $i$ - th state and $k_{B}$ is the Boltzmann constant, and the microscopic entropy of system in the $i-t h$ state is

$$
S^{i}=\sum_{j=1}^{N_{i}} S_{j}^{i}=-k_{B} \sum_{j=1}^{N_{i}} \ln \left(\omega_{j}^{i} p_{j}^{i}\right)
$$

the macroscopic entropy of system is a statistical average value of its microscopic entropy. The macroscopic entropy $S$ is

$$
S=\sum_{i=1}^{M} P_{i} S^{i}=-k_{B} \sum_{i=1}^{M} \sum_{j=1}^{N_{i}} P_{i} \ln \left(\omega_{j}^{i} p_{j}^{i}\right),
$$

substituting Eqs. (3), (5) and (8) into (1), we have

$$
\sum_{i=1}^{M} \sum_{j=1}^{N_{i}} P^{i} f_{j}^{i}=\sum_{i=1}^{M} P_{i}\left(\sum_{j=1}^{N_{i}}\left(T_{j}^{i}+V_{j}^{i}\right)+\sum_{j, m}^{N_{i}} V_{j m}^{i}\right)+T k_{B} \sum_{i=1}^{M} \sum_{j=1}^{N_{i}} P_{i} \ln \left(\omega_{j}^{i} p_{j}^{i}\right),
$$

Eq. (9) is the macroscopic free energy equation of system. Deleting the sum mark $\sum_{i=1}^{M} P_{i}$, we have

$$
F^{i}=\sum_{j=1}^{N_{i}} f_{j}^{i}=\sum_{j=1}^{N_{i}}\left(T_{j}^{i}+V_{j}^{i}\right)+\sum_{j, m}^{N_{i}} V_{j m}^{i}+T k_{B} \sum_{j=1}^{N_{i}} \ln \left(\omega_{j}^{i} P_{j}^{i}\right)
$$


Eq. (10) is the system's microscopic free energy equation in the $i-t h$ state. Deleting the sum mark $\sum_{j=1}^{N_{i}}{ }^{3}$, there is

$$
f_{j}^{i}=T_{j}^{i}+V_{j}^{i}+T k_{B} \ln \left(\omega_{j}^{i} p_{j}^{i}\right)
$$

Eq. (11) is the free energy equation of the $j-t h$ single particle in the $i-t h$ microscopic state. Deleting the suffix $j$, we obtain the free energy equation of arbitrary particle in $i-t h$ microscopic state, it is

$$
f^{i}=T^{i}+V^{i}+T k_{B} \ln \left(\omega^{i} p^{i}\right) .
$$

\section{Non-relativistic quantum theory at finite temperature}

In section 2, we give the free energy equation of a single particle and multiple particles system in the $i$ - th state, they are shown in Eqs. (10) and (12). Quantizing the Eqs. (10) and (12), we can obtain the finite temperature quantum wave equation of single particle and multiple particles. Making the mechanical quantities in Eq. (12) become the operator:

$$
\left\{\begin{array}{l}
\hat{f}^{i}=i \hbar \frac{\partial}{\partial t} \\
\hat{T}^{i}=-\frac{\hbar^{2}}{2 m} \nabla^{2} \\
\hat{V}^{i}=V
\end{array}\right.
$$

we have

$$
i \hbar \frac{\partial}{\partial t} \psi_{i}(\vec{r}, t, T)=\left[-\frac{\hbar^{2}}{2 m} \nabla^{2}+V+T k_{B} \ln \left(\omega^{i} p^{i}\right)\right] \psi_{i}(\vec{r}, t, T),
$$

where $\psi_{i}(\vec{r}, t, T)$ is the $i-t h$ state wave function. Eq. (14) is the time-dependent and temperature-dependent quantum wave equation at finite temperature for a single particle in the $i-t h$ state, which is different from the zero temperature quantum wave equation, i.e., Schrodinger equation. By the method of separation variable

$$
\psi_{i}(\vec{r}, t, T)=\psi_{i}(\vec{r}) \phi(T) f(t)
$$

substituting Eq. (15) into (14), there are

$$
\begin{gathered}
i \hbar \frac{d f(t)}{d t}=E_{i}(T) f(t), \\
{\left[-\frac{\hbar^{2}}{2 m} \nabla^{2}+V\right] \psi_{i}(\vec{r})=E_{i}(0) \psi_{i}(\vec{r}),} \\
T k_{B} \ln \left(\omega^{i} p^{i}\right) \phi(T)=E_{i}^{\prime}(T) \phi(T),
\end{gathered}
$$

and

$$
E_{i}(T)=E_{i}(0)+E_{i}^{\prime}(T)=E_{i}(0)+T k_{B} \ln \left(\omega^{i} p^{i}\right)
$$

where $E_{i}(0)$ is the eigenvalue of Schrodinger equation (17) in the $i-t h$ state. The solution of Eq. (16) is

$$
f(t)=e^{-\frac{i}{\hbar} E_{i}(T) t}
$$

substituting Eq. (20) into (14), there is

$$
\left[-\frac{\hbar^{2}}{2 m} \nabla^{2}+V+T k_{B} \ln \left(\omega^{i} p^{i}\right)\right] \psi_{i}(\vec{r}, T)=E_{i}(T) \psi_{i}(\vec{r}, T)
$$


where $\psi_{i}(\vec{r}, T)=\psi_{i}(\vec{r}) \phi(T), E_{i}(T)$ and $p^{i}$ are corresponding to the eigenfunction, eigenvalues, and probability in the $i-t h$ state. Eq. (21) is the time-independent and temperature-dependent quantum wave equation. When the temperature $T=0$, Eq. (21) becomes Schrodinger equation.

The probability $p^{i}$ is

$$
p^{i}=\frac{1}{Z(T)} e^{-\beta E_{i}},
$$

where $Z(T)=\sum_{i} \omega_{i} e^{-\beta E_{i}}$, and $\beta=\frac{1}{k_{B} T}$.

For Eq. (19), there are two situation:

(1) When $T=0$

$$
E_{i}(T)=E_{i}(0)
$$

(2) When $T \neq 0$, substituting Eq. (22) into (19), we have

$$
\begin{aligned}
E_{i}(T) & =E_{i}(0)+T k_{B} \ln \left(\omega^{i} p^{i}\right) \\
& =E_{i}(0)-E_{i}(T)+T k_{B} \ln \omega^{i}-T k_{B} \ln Z(T),
\end{aligned}
$$

i.e.,

$$
E_{i}(T)=\frac{1}{2} E_{i}(0)+\frac{1}{2} T k_{B} \ln \omega^{i}-T k_{B} \ln \sqrt{Z(T)},
$$

For a dimensional harmonic oscillator, $\omega^{i}=1$, we have

$$
E_{i}(T)=\frac{1}{2} E_{i}(0)-T k_{B} \ln \sqrt{Z(T)},
$$

where

$$
E_{i}(0)=\left(i+\frac{1}{2}\right) \hbar \omega
$$

and

$$
\begin{aligned}
Z(T) & =\sum_{i} e^{-\beta E_{i}(T)} \\
& =\sum_{i} e^{-\frac{1}{k_{B} T}\left(\frac{1}{2} E_{i}(0)-T k_{B} \ln \sqrt{Z(T)}\right)} \\
& =\sum_{i} e^{-\frac{1}{2 k_{B} T} E_{i}(0)} \cdot \sqrt{Z(T)},
\end{aligned}
$$

to get

$$
\begin{aligned}
\sqrt{Z(T)} & =\sum_{i} e^{-\frac{1}{2 k_{B} T} E_{i}(0)} \\
& =\frac{e^{-\frac{\hbar \omega}{4 k_{B} T}}}{1-e^{-\frac{\hbar \omega}{2 k_{B} T}}}
\end{aligned}
$$

substituting Eqs. (27) and (29)into (26), we obtain

$$
E_{i}(T)=\frac{i+1}{2} \hbar \omega+k_{B} T \ln \left(1-e^{-\frac{\hbar \omega}{2 k_{B} T}}\right) .
$$

Eq. (30) is the $i-t h$ energy level of a dimensional harmonic oscillator at finite temperature. Its energy level at zero temperature and finite temperature can be written as

$$
E_{n}= \begin{cases}\left(n+\frac{1}{2}\right) \hbar \omega & (T=0) \\ \frac{1}{2}(n+1) \hbar \omega+k_{B} T \ln \left(1-e^{-\frac{\hbar \omega}{2 k_{B} T}}\right) & (T \neq 0)\end{cases}
$$


From Eq. (25), we have

$$
2\left(E_{i}\left(T_{1}\right)-E_{j}\left(T_{1}\right)\right)=E_{i}(0)-E_{j}(0)+T_{1} k_{B} \ln \frac{\omega^{i}}{\omega^{j}}
$$

and

$$
2\left(E_{i}\left(T_{2}\right)-E_{j}\left(T_{2}\right)\right)=E_{i}(0)-E_{j}(0)+T_{2} k_{B} \ln \frac{\omega^{i}}{\omega^{j}}
$$

Eq. (32) minus (33), there is

$$
\nu_{i j}\left(T_{1}-T_{2}\right)=\nu_{i j}\left(T_{1}\right)-\nu_{i j}\left(T_{2}\right)=\frac{k_{B}}{2 h}\left(T_{1}-T_{2}\right) \ln \frac{\omega^{i}}{\omega^{j}}
$$

where $\nu_{i j}(T)=\left(E_{i}(T)-E_{j}(T)\right) / h$, which is the transition frequency from the $i-t h$ state to the $j-t h$ state.

From Eq. (34), we can obtain the results: (1) when $\omega^{i}=\omega^{j}, \nu_{i j}\left(T_{1}-T_{2}\right)=0$, i.e., when the degeneracies of two energy levels are the same, the transition frequency is unchanged with different temperature. (2) When $T_{1} \neq T_{2}$ and $\omega^{i} \neq \omega^{j}, \nu_{i j}\left(T_{1}-T_{2}\right)$ is direct proportion to $T_{1}-T_{2}$.

The time-dependent and temperature-dependent wave function at $i-t h$ state is

$$
\begin{aligned}
\psi_{i}(\vec{r}, t, T) & =\psi_{i}(\vec{r}) \phi(T) f(t) \\
& =\psi_{i}(\vec{r}) \phi(T) e^{-i \frac{E_{i}(T)}{\hbar} t} \\
& =\psi_{i}(\vec{r}) \phi(T) e^{-\frac{i}{2 \hbar} E_{i}(0) t} \cdot e^{-\frac{i}{2 \hbar} T k_{B} \ln \frac{\omega^{i}}{Z(T)}},
\end{aligned}
$$

quantizing Eq. (10), we have

$$
i \hbar \frac{\partial}{\partial t} \psi_{i}\left(\vec{r}_{1}, \vec{r}_{2}, \ldots \vec{r}_{N_{i}}, t, T\right)=\left[\sum_{j=1}^{N_{i}}\left(-\frac{\hbar^{2}}{2 m_{j}} \nabla_{j}^{2}+V_{j}\right)+\sum_{j, m}^{N_{i}} V_{j m}+k_{B} T \sum_{j=1}^{N_{i}} \ln \left(\omega_{j}^{i} p_{j}^{i}\right)\right] \psi_{i}\left(\vec{r}_{1}, \vec{r}_{2}, \ldots \vec{r}_{N_{i}}, t, T\right)
$$

with the identity principle, there is

$$
p_{1}^{i}=p_{2}^{i}=\cdots=p_{j}^{i}=\cdots=p_{N_{i}}^{i}=p^{i}
$$

Eq. (37) becomes

$$
i \hbar \frac{\partial}{\partial_{t}} \psi_{i}\left(\vec{r}_{1}, \vec{r}_{2}, \ldots \vec{r}_{N_{i}}, t, T\right)=\left[\sum_{j=1}^{N_{i}}\left(-\frac{\hbar^{2}}{2 m_{j}} \nabla_{j}^{2}+V_{j}\right)+\sum_{j m}^{N_{i}} V_{j m}+k_{B} T \sum_{j=1}^{N_{i}} \ln \left(\omega_{j}^{i} p^{i}\right)\right] \psi_{i}\left(\vec{r}_{1}, \vec{r}_{2}, \ldots \vec{r}_{N_{i}}, t, T\right)
$$

Eq. (38) is the finite temperature quantum theory of multiple particles.

\section{Lagrangian function at finite temperature}

For the finite temperature quantum equation (14), its complex conjugate equation is

$$
-i \hbar \frac{\partial}{\partial t} \psi_{i}^{*}(\vec{r}, t, T)=\left[-\frac{\hbar^{2}}{2 m} \nabla^{2}+V+T k_{B} \ln \left(\omega^{i} p^{i}\right)\right] \psi_{i}^{*}(\vec{r}, t, T),
$$

the Lagrangian function of the finite temperature quantum equations (14) and (39) can be taken as

$$
L_{i}=i \hbar \psi_{i}^{*} \cdot \dot{\psi}_{i}-\frac{\hbar^{2}}{2 m} \nabla \psi_{i}^{*} \cdot \nabla \psi_{i}-V \psi_{i}^{*} \psi_{i}-T k_{B} \ln \left(\omega^{i} p^{i}\right) \psi_{i}^{*} \cdot \psi_{i}
$$

where $L_{i}$ is the Lagrangian function of the $i-t h$ quantum state.

From Eq. (40), we have

$$
\frac{\partial L_{i}}{\partial \psi_{i}}=-V \psi_{i}^{*}-T k_{B} \ln \left(\omega^{i} p^{i}\right) \psi_{i}^{*}
$$




$$
\begin{gathered}
\frac{\partial L_{i}}{\partial \dot{\psi}_{i}}=i \hbar \psi_{i}^{*}, \\
\frac{\partial L_{i}}{\partial\left(\frac{\partial \psi_{i}}{\partial x_{i}}\right)}=-\frac{\hbar^{2}}{2 m} \frac{\partial \psi_{i}^{*}}{\partial x_{i}}, \\
\frac{\partial L_{i}}{\partial \psi_{i}^{*}}=i \hbar \dot{\psi}_{i}-V \psi_{i}-T k_{B} \ln \left(\omega^{i} p^{i}\right) \psi_{i}, \\
\frac{\partial L_{i}}{\partial \dot{\psi}_{i}^{*}}=0
\end{gathered}
$$

and

$$
\frac{\partial L_{i}}{\partial\left(\frac{\partial \psi_{i}^{*}}{\partial x_{i}}\right)}=-\frac{\hbar^{2}}{2 m} \frac{\partial \psi_{i}}{\partial x_{i}}
$$

substituting Eqs. (41)-(43) into Lagrangian equation

$$
\frac{\partial L_{i}}{\partial \psi_{i}^{*}}-\frac{\partial}{\partial t}\left(\frac{\partial L_{i}}{\partial \dot{\psi}_{i}^{*}}\right)-\sum_{i=1}^{3} \frac{\partial}{\partial x_{i}}\left(\frac{\partial L_{i}}{\partial\left(\frac{\partial \psi_{i}^{*}}{\partial x_{i}}\right)}\right)=0,
$$

to get

$$
i \hbar \dot{\psi}_{i}-V \psi_{i}-T k_{B} \ln \left(\omega^{i} p^{i}\right) \psi_{i}+\frac{\hbar^{2}}{2 m} \sum_{i=1}^{3} \frac{\partial^{2} \psi_{i}}{\partial x_{i}^{2}}=0 .
$$

Eq. (48) is equation (14)

$$
i \hbar \frac{\partial}{\partial t} \psi_{i}(\vec{r}, t, T)=\left[-\frac{\hbar^{2}}{2 m} \nabla^{2}+V+T k_{B} \ln \left(\omega^{i} p^{i}\right)\right] \psi_{i}(\vec{r}, t, T),
$$

substituting Eqs. (44)-(46) into Lagrangian equation

$$
\frac{\partial L_{i}}{\partial \psi_{i}}-\frac{\partial}{\partial t}\left(\frac{\partial L_{i}}{\partial \dot{\psi}_{i}}\right)-\sum_{i=1}^{3} \frac{\partial}{\partial x_{i}}\left(\frac{\partial L_{i}}{\partial\left(\frac{\partial \psi_{i}}{\partial x_{i}}\right)}\right)=0,
$$

to get

$$
-V \psi_{i}^{*}-T k_{B} \ln \left(\omega^{i} p^{i}\right) \psi_{i}^{*}-i \hbar \dot{\psi}_{i}^{*}+\sum_{i=1}^{3} \frac{\hbar^{2}}{2 m} \frac{\partial^{2} \psi_{i}^{*}}{\partial x_{i}^{2}}=0,
$$

Eq. (51) is equation (39)

$$
-i \hbar \frac{\partial}{\partial t} \psi_{i}^{*}(\vec{r}, t, T)=\left[-\frac{\hbar^{2}}{2 m} \nabla^{2}+V+T k_{B} \ln \left(\omega^{i} p^{i}\right)\right] \psi_{i}^{*}(\vec{r}, t, T) .
$$

When the finite temperature Lagrangian function is taken as the form of equation (40), we can obtain the finite temperature quantum wave equations (14) and (39). 


\section{Conclusion}

With the thermodynamic and statistical mechanics, we give the microscopic free energy of a single particle and multiple particles system. By quantization, we give the non-relativistic finite temperature quantum wave equation for a single particle and multiple particles, and give the relation between energy eigenvalues, eigenfunctions, transition frequency and temperature. Otherwise, we give the relation between transition frequency and temperature and obtain some results: (1) when $\omega^{i}=\omega^{j}, \nu_{i j}\left(T_{1}-T_{2}\right)=0$, i.e., when the degeneracies of two energy levels are the same, the transition frequency is unchanged with different temperature. (2) When $T_{1} \neq T_{2}$ and $\omega^{i} \neq \omega^{j}$, the variance of transition frequency $\nu_{i j}\left(T_{1}-T_{2}\right)$ is direct proportion to $T_{1}-T_{2}$. The finite temperature quantum theory should be tested by experiment in the future, and they can be studied superconductivity mechanism and Bose-Einstein Condensate and so on.

1 T. Matsubra, Prog. Theor. Phys. 14, 351 (1955).

2 E. S. Fradkin, Doklady Akad. Nauk. 98 (1954) 47; ibid. 100, 897 (1955).

3 C. W. Bernard, Phys. Rev. D9, 3312 (1974).

4 Fetter A L and Walecka J D 1971 Quantum Theory of Many Particle Systems (New York: McGraw-Hill).

${ }^{5}$ L. Brillouin, Comptes Rendus 183, 24 (1926).

${ }^{6}$ H. A. Kramers, Z. Phys. 39, 828 (1926).

7 G. Wentzel, Z. Phys. 38, 518 (1926).

8 C. W. Bernard, Phys. Rev. D 9, 3312 (1974).

9 L. Dolan and R. Jackiw, Phys. Rev. D 9, 3320 (1974).

10 S. Weinberg, Phys. Rev. D 9, 3357 (1974).

11 R. F. Dashen, Shang-keng Ma and R. Rajaraman, Phys. Rev. D 11, 1499 (1975).

12 B. J. Harrington, Phys. Rev. D 18, 2982 (1978).

13 L. Dolan and J. Kiskis, Phys. Rev. D 20, 505 (1979). 